DFTT 37/93

August 1993

\title{
On the Neutralino as Dark Matter Candidate. I. Relic Abundance.
}

\author{
A. Bottino, V. de Alfaro, N. Fornengo, G. Mignola, M. Pignone \\ Dipartimento di Fisica Teorica dell'Università di Torino \\ and INFN, Sezione di Torino, Italy \\ Via P. Giuria 1, 10125 Torino, Italy
}

\begin{abstract}
The neutralino relic abundance is evaluated for a wide range of the neutralino mass, $20 \mathrm{GeV} \leq m_{\chi} \leq 1 \mathrm{TeV}$, by taking into account the full set of final states in the neutralino-neutralino annihilation. The analysis is performed in the Minimal SuSy Standard Model; it is not restricted by stringent GUT assumptions but only constrained by present experimental bounds. We also discuss phenomenological aspects which are employed in the companion paper (II. Direct Detection) where the chances for a successful search for dark matter neutralino are investigated.
\end{abstract}




\section{Introduction.}

The general features of cosmological structures, as they are observed and understood at present, lead to the conclusion that large amount of the matter in our Universe is in the form of Cold Dark Matter (CDM). This circumstance has recently prompted new detailed investigations about the neutralino $(\chi)$, since this SuSy particle appears to be the most favorite candidate for CDM. Here we analyse one of its basic properties, the relic abundance, by extending a previous investigation of ours [1] (which was confined to neutralino masses below the $\mathrm{W}$-boson mass) to a much wider range of neutralino masses: $20 \mathrm{GeV} \leq m_{\chi} \leq 1 \mathrm{TeV}$ for the most general neutralino composition. Furthermore we take into account the whole set of exchange diagrams and final states in the $\chi-\chi$ annihilation process which is the fundamental ingredient in the evaluation of the relic abundance. In addition, radiative corrections to the Higgs boson masses as well as to the relevant coupling constants are appropriately included in our evaluations.

In the present investigation the theoretical framework is represented by the Minimal Supersymmetric Standard Model (MSSM); only one standard GUT assumption is employed with the purpose of simplifying the phenomenological discussion.

The analysis presented here differs from the previous ones [2-8] in at least one of the features mentioned above. In particular the most recent analyses on this subject by other authors are mainly based upon GUT schemes which also include supergravity; this automatically implies model-dependent relationships between the various masses which come into play. Here we prefer to consider a more flexible scheme where unknown masses are not a priori fixed, but are only constrained by present experimental bounds.

We also wish to emphasize that we do not restrain our attention to regions of the parameter space where the neutralino would provide by itself the total amount of required CDM, but we rather widely explore the parameter space with the aim of investigating the chances to detect the neutralino as a dark matter candidate by direct or indirect searches. In fact regions where the detection event rates are higher do not necessarily coincide with the locations in parameter space where the relic density is larger, since stronger coupling of the neutralino with matter may compensate for partial depletion in the neutralino local density. Indeed actual detection of dark matter neutralino would be an achievement of paramount interest even if the neutralino does not exhaust our need for CDM. For these reasons, whereas in the present paper (hereafter called I) we discuss the neutralino relic abundance, in the companion paper (II) which follows this one, we analyse the problem 
of its direct detection. The way of presenting our results in paper I is mainly shaped according to the needs for the applications discussed in paper II.

\section{Minimal SuSy Standard Model.}

Our theoretical framework is the MSSM, with the standard definition of neutralino as the lowest-mass linear combination of photino, zino and higgsinos,

$$
\chi=a_{1} \tilde{\gamma}+a_{2} \tilde{Z}+a_{3} \tilde{H}_{1}^{0}+a_{4} \tilde{H}_{2}^{0}
$$

where $\tilde{\gamma}$ and $\tilde{Z}$ are linear combinations of the $\mathrm{U}(1)$ and $\mathrm{SU}(2)$ neutral gauginos, $\tilde{B}$ and $\tilde{W}_{3}$,

$$
\begin{aligned}
& \tilde{\gamma}=\cos \theta_{W} \tilde{B}+\sin \theta_{W} \tilde{W}_{3}, \\
& \tilde{Z}=-\sin \theta_{W} \tilde{B}+\cos \theta_{W} \tilde{W}_{3},
\end{aligned}
$$

$\theta_{W}$ being the Weinberg angle. As usual, $\chi$ is assumed to be the lightest supersymmetric particle (LSP) and then stable if R-parity is conserved. Its mass as well as its composition depend on the parameters: $M_{1}, M_{2}$ (masses of $\tilde{B}$ and of $\tilde{W}_{3}$, respectively), $\mu$ (Higgs mixing parameter) and $\tan \beta=v_{u} / v_{d}\left(v_{u}\right.$ and $v_{d}$ being the v.e.v.'s which give masses to up-type and down-type quarks). A standard procedure in current literature is to embed the MSSM in GUT, so that a relationship between $M_{1}$ and $M_{2}$ follows: $M_{1}=5 / 3 \tan ^{2} \theta_{W} \simeq 0.5 M_{2}$. Relaxing this assumption may modify some neutralino properties in a significant way; this has been discussed by some authors in the restricted case $m_{\chi}<m_{W}[9,10]$.

In this paper we will report our results under the assumption that the GUT-induced relation between $M_{1}$ and $M_{2}$ holds, as this is a most natural hypothesis. Extensions of the present analysis due to the relaxation of this relationship will be presented in a forthcoming paper [11].

As mentioned above, in the present analysis also high values of $m_{\chi}$ are considered (up to $1 \mathrm{TeV}$ ). Thus, in order to deal with a parameter space large enough to contain various compositions for $\chi$ at any value of $m_{\chi}$, we have taken wide ranges for $M_{2}$ and $\mu: 20 \mathrm{GeV} \leq M_{2} \leq 6 \mathrm{TeV}, 20 \mathrm{GeV} \leq|\mu| \leq$ $3 \mathrm{TeV}$. The scatter plots which are presented in section 3 are obtained by varying $M_{2}$ and $\mu$ in these ranges. As far as $\tan \beta$ is concerned, in order to cover a wide range for it we will representatively choose the following values: $\tan \beta=2, \tan \beta=8$ (or rather the range $6 \leq \tan \beta \leq 10$ in a number of 
extended scatter plots) and $\tan \beta=20$. It is worth noticing that the ranges chosen for the parameters automatically disregard regions of the parameters space which have been excluded by LEP.

To illustrate the general features of the neutralino we give in Fig.1 a representation of its mass and composition in the $M_{2}-\mu$ plane at $\tan \beta=8$. This figure clearly shows that the neutralino composition tends to be very pure (either pure higgsino or pure gaugino, depending on whether $M_{2}>2|\mu|$ or $\left.M_{2}<2|\mu|\right)$ as $M_{2}$ and $\mu$ increase. This property, already discussed in previous works, is due to the fact that in the neutralino $4 \times 4$ mass matrix the higgsino sector asymptotically decouples from the gaugino sector as $M_{2}$, $|\mu| \gg M_{Z}$. Before this asymptotic regime turns on, and so for $M_{2}, 2|\mu| \lesssim$ a few hundreds $\mathrm{GeV}$ (neutralino masses $\lesssim 100 \mathrm{GeV}$ ), mixed higgsino-gaugino configurations are important. This property plays a crucial role in some of the conclusions we will draw about the neutralino relic abundance and the detection event rates.

Effective interaction properties of the neutralino depend sensitively on the three physical neutral Higgs bosons of the MSSM: the two CP-even bosons: h,H (of masses $m_{h}, m_{H}$ with $m_{H}>m_{h}$ ) and the CP-odd one: A (of mass $m_{A}$ ). Radiatively corrected relationships between these masses are used here. Thus, taking $m_{h}$ as an independent parameter, $m_{A}$ and $m_{H}$ are functions of $m_{h}, \tan \beta, m_{t}$ (mass of the top quark) and $\tilde{m}$ (mass of the top scalar partners, taken as degenerate). To represent our results we will take two values for $m_{h}: m_{h}=50 \mathrm{GeV}$ (which entails $m_{A} \sim 50 \mathrm{GeV}$ ) and $m_{h}=80 \mathrm{GeV}$ (this implies $m_{A}=83 \mathrm{GeV}$ for $\tan \beta=8$ and $m_{A}=170 \mathrm{GeV}$ for $\tan \beta=2$ ). The top quark mass has been taken at the value $m_{t}=150$ $\mathrm{GeV}$. The sfermion masses will be discussed in the next section.

\section{Relic Abundance.}

The neutralino relic abundance $\Omega_{\chi} h^{2}(\Omega$ is the density parameter of the Universe: $\left.\Omega=\rho / \rho_{c}, \rho_{c}=1.88 \times 10^{-29} h^{2} \mathrm{~g} \mathrm{~cm}^{-3}, 0.4 \leq h \leq 1\right)$ has been evaluated employing the usual formula [12]

$$
\Omega_{\chi} h^{2}=2.13 \times 10^{-11}\left(\frac{T_{\chi}}{T_{\gamma}}\right)^{3}\left(\frac{T_{\gamma}}{2.7 K}\right)^{3} N_{F}^{1 / 2}\left(\frac{\mathrm{GeV}^{-2}}{a x_{f}+\frac{1}{2} b x_{f}^{2}}\right)
$$

Here $T_{\gamma}$ is the present temperature of the microwave background, $T_{\chi} / T_{\gamma}$ is the reheating factor of the photon temperature as compared to the neutralino 
temperature, $x_{f}=T_{f} / m_{\chi} \simeq 1 / 20$ where $T_{f}$ is the neutralino freeze-out temperature. The $x_{f}$-dependent expression in the denominator of Eq.(3.1) represents the integration from $T_{f}$ down to the present temperature of the thermally averaged quantity $\left\langle\sigma_{a n n} v>=a+b x\right.$ where $\sigma_{a n n}$ is the $\chi-\chi$ annihilation cross-section, $v$ the relative velocity and $x=T / m_{\chi}$. In the evaluation of the annihilation cross-section we have considered the whole set of final states: 1) fermion-antifermion pair, 2) pair of neutral Higgs bosons, 3) pair of charged Higgs bosons, 4) one Higgs boson-one gauge boson, 5) pair of gauge bosons $\left(W^{+} W^{-}, Z Z\right)$. For the final state 1), the following diagrams have been considered: Higgs-exchange diagrams and $\mathrm{Z}$-exchange diagram in the s-channel, $\tilde{f}$-exchange diagrams in the $\mathrm{t}$-channel. For the final states 2-5) we have taken Higgs-exchange and Z-exchange diagrams in the s-channel, and either neutralinos (the full set of the four mass eigenstates) or chargino exchange in the $\mathrm{t}$-channel, depending on the electric charges of the final particles. As for the sfermion masses, we have considered two extreme cases: one with mass values as low as possible, compatibly with the present experimental bound and with the assumption that $\chi$ is the LSP, and a second one where all sfermions are very massive. The lower limit that we have conservatively used for the sfermion masses is the LEP bound of 45 $\mathrm{GeV}$, since the CDF limit [13] on the squark masses does not appear to be consistent with a massive neutralino [14].

Thus we have considered the two cases: 1) $m_{\tilde{f}}=1.2 m_{\chi}$, when $m_{\chi}>45$ $\mathrm{GeV} ; m_{\tilde{f}}=45 \mathrm{GeV}$ otherwise, except for the mass of the top scalar partner (the only one relevant to radiative corrections) which has been taken $\tilde{m}=3$ $\mathrm{TeV} ; 2) m_{\tilde{f}}=3 \mathrm{TeV}$ for all sfermions.

Let us now turn to the presentation of our results. In Fig.s 2-3 we report, in the $M_{2}-\mu$ plane, the dominance of various final states at $\tan \beta=8$, $m_{h}=50 \mathrm{GeV}$ and for the two representative values for $m_{\tilde{f}}$. Dominance of a particular final state means that this channel weighs for at least a factor of 5 over the other states in the quantity $\left\langle\sigma_{a n n} v>\right.$. Fig. 2, which refers to small values of $m_{\tilde{f}}$, shows that in large regions of the $M_{2}, \mu$ plane the $f \bar{f}$ and the two gauge bosons final states dominate. However it has to be stressed that for $\mu>0$ in a significant region of the parameter space, where mixed gaugino-higgsino configurations occur, there is dominance of one Higgs boson-one gauge boson final state. This feature was absent in Ref. [3], where this contribution was estimated to be subdominant. The one Higgs boson-one gauge boson final states are even more important when all the $m_{\tilde{f}}$ masses are set at $3 \mathrm{TeV}$ (see Fig. 3 ); in fact, a drastic increase in $m_{\tilde{f}}$ has the obvious consequence of a suppression of the $\tilde{f}$-exchange amplitude, and this significantly depresses the $f \bar{f}$ final states. On the contrary, dominance 
of the two Higgs boson final states is limited to a few isolated points in the parameter space, as is illustrated in Fig.s $2-3$. These general features remain almost unaltered if we move from $\tan \beta=8$ to smaller values, $\tan \beta \sim 2$.

Let us now discuss the neutralino relic abundance. In Fig. 4 we report our results in the form of scatter plots obtained by varying $M_{2}, \mu$ in the parameter space previously defined and by varying $\tan \beta$ in the range $6 \leq$ $\tan \beta \leq 10 . \Omega_{\chi} h^{2}$ versus $m_{\chi}$ is shown for three different types of neutralino compositions: higgsino dominance (dominance here means $90 \%$ or more), gaugino dominance, maximal higgsino-gaugino mixing (i.e., $0.45 \leq a_{1}^{2}+a_{2}^{2} \leq$ 0.55). In Fig. 4a, (higgsino dominance) some characteristic features are very clearly displayed. A number of pronounced dips (and of sharp falls off) in $\Omega_{\chi} h^{2}$ reflect the presence of poles (and the opening of new thresholds) in the annihilation cross section. In sequence we have: at $m_{\chi} \sim 25 \mathrm{GeV}$ the $h$ and the $A$ poles, at $m_{\chi} \sim 45 \mathrm{GeV}$ the $Z$ pole, at $m_{\chi} \sim 90 \mathrm{GeV}$ the threshold for the $\chi-\chi$ annihilation into channels $W^{+} W^{-}$and $Z Z$. In the case of gaugino dominance (see Fig.4b) sfermion-exchange amplitudes provide large contributions to the annihilation cross section, with the effect of depressing the neutralino relic abundance as compared to the case of higgsino dominance for $m_{\chi} \lesssim 90 \mathrm{GeV}$. At higher $m_{\chi}$ values the gaugino-dominated compositions give a larger $\Omega_{\chi} h^{2}$, since here the $f \bar{f}$ final state is dominant, but somewhat hampered by the running values of the $\tilde{f}$ mass: $m_{\tilde{f}}=1.2 m_{\chi}$. Compositions with large mixings (see Fig.4c-d) entail rather low values of $\Omega_{\chi} h^{2}$, due to the substantial contribution to the annihilation provided by Higgs-exchange and $\tilde{f}$-exchange. Nevertheless, these neutralino configurations contribute significantly to the event rates for direct neutralino search (as discussed in paper II). The previous discussion should make the features of Fig.5 quite transparent. In fact here high values for $m_{\tilde{f}}$ force the neutralino relic density of the gaugino-dominated configurations to be large, by inhibiting the $\tilde{f}-$ exchange amplitude. This same mechanism is the reason for the depletion in the $f \bar{f}$ final state dominance that we notice in the plot of Fig.3 when we compare it with the one in Fig.2.

Fig.s $6-7$ show the neutralino relic abundance when $\tan \beta$ is small: $\left(\tan \beta=2\right.$, for definiteness) and $m_{h}=80 \mathrm{GeV}$. Higgsino-dominated configurations at small $m_{\chi}$ (below the thresholds for $W^{+} W^{-}$and $Z Z$ ) display large values of $\Omega_{\chi} h^{2}$, since now, smaller values of $\tan \beta$ and larger values of Higgs boson masses, both have the effect of suppressing the annihilation channels with Higgs exchanges. As for the gaugino-dominated compositions it is worth noticing that in the case of large $m_{\tilde{f}}$ (see Fig.7b) $\Omega_{\chi} h^{2}$ displays a behaviour which is rather common in the context of some supergravity 
inspired models. In fact in these schemes it frequently occurs that theoretical and phenomenological constraints restrict $\tan \beta$ to very small values and sfermion masses to high values, with the consequence that the cosmological requirement $\Omega_{\chi} h^{2}<1$ can only be met at the Higgs-poles or at the $\mathrm{Z}$-pole. Consequently, particular care has to be taken in the evaluation of the relic abundance [15] in these models. In our kind of analysis, fine-tuning of $m_{\chi}$ with the masses of the Higgs bosons or of the gauge bosons is not required and would then appear rather accidental.

A final scatter plot for $\Omega_{\chi} h^{2}$ is shown in Fig. 8 for $\tan \beta=20$ and $m_{h}=$ $50 \mathrm{GeV}$.

A word of warning is required about the effects due to the possible occurrence of an approximate degeneracy (within $\sim 15 \%$ ) between the neutralino and some other SuSy particle. When this happens the neutralino decoupling mechanism is enhanced due to the annihilation process involving the neutralino with the other SuSy particle which is close to it in mass (this process is usually denoted as coannihilation in the literature) $[15,16]$. Effects on $\Omega_{\chi} h^{2}$ due to coannihilation may be large (one order of magnitude or more, depending on the nature of the coannihilating particle and on other details of the theoretical scheme). Apart from the peculiar and accidental case when for instance a sfermion and the $\chi$ would almost have the same mass, a natural case of approximate degeneracy occurs in the neutralino-chargino sector. However, this happens in regions of the parameter space of higgsino dominance. In paper II it is shown that chances of detecting dark matter neutralinos rely essentially on mixed or gaugino compositions for neutralinos. Thus coannihilation does not significantly affect the evaluations of the event rates presented in paper II.

In conclusion our analysis confirms that the neutralino, even at mass values higher than the $\mathrm{W}$-mass, may satisfy the attributes required for a good candidate for CDM. It is also clear that the theoretical evaluations for neutralino relic abundance only suffer from the lack of information about some of the particles that naturally come into play, such as the Higgs bosons and any SuSy object. Only new experimental inputs can help theory in sharpening its predictions for neutralino dark matter.

This work was supported in part by Research Funds of the Ministero dell'Università e della Ricerca Scientifica e Tecnologica. 


\section{References}

[1] A.Bottino, V.de Alfaro, N.Fornengo, G.Mignola and S.Scopel, Astroparticle Phys. 1(1992)61.

[2] References where $m_{\chi}$ beyond the $\mathrm{W}$-mass has been considered are given in [3-8]. For other papers see, for instance, the references quoted in [1].

[3] K.Griest, M.Kamionkowski and M.S.Turner, Phys. Rev. D41(1990)3565

[4] J.McDonald, K.A.Olive and M.Srednicki, Phys. lett. B283(1992)80.

[5] P.Gondolo, M.Olechowski and S.Pokorski, MPI-Ph/92-81 preprint.

[6] M.Drees and M.M.Nojiri, Phys. Rev. D47(1993)376.

[7] P.Nath and R.Arnowitt, CTP-TAMU-66/92 preprint;

J.L.Lopez, D.V.Nanopoulos, and K.Yuan, CTP-TAMU-14/93 preprint.

[8] R.G.Roberts and L.Roszkowski, Phys. Lett. B309(1993)329.

[9] K.Griest and L.Roszkowski, Phys. Rev. D46(1992)3309.

[10] S.Mizuta, D.Ng and M.Yamaguchi, Phys. Lett. B300(1993)96.

[11] A.Bottino, V.de Alfaro, N.Fornengo, G.Mignola and M.Pignone, to appear.

[12] J.Ellis, J.S.Hagelin, D.V.Nanopoulos, K.Olive and M.Srednicki, Nucl. Phys. B238(1984)453.

[13] F.Abe et al. (CDF Collaboration), Phys. Rev. Lett. 69(1992)3439.

[14] see also H.Baer, X.Tata and J.Woodside, Phys. Rev. D44(1991)207 for a discussion on cascade decays whose inclusion weakens the bounds of Ref.[13].

[15] K.Griest and D.Seckel, Phys. Rev. D43(1991)3191.

[16] S.Mizuta and M.Yamaguchi, Phys. Lett. B298(1993)120. 


\section{Figure Captions}

Figure 1. Isomass curves and composition lines for neutralino in the $M_{2}-\mu$ plane for $\tan \beta=8$. Dashed lines are lines of constant $\chi$ mass $\left(m_{\chi}\right.$ $=30 \mathrm{GeV}, 100 \mathrm{GeV}, 300 \mathrm{GeV}$ and $1 \mathrm{TeV}$ ). Solid lines refer to constant gaugino fraction $f_{g}$ in the neutralino composition $\left(f_{g}=a_{1}^{2}+a_{2}^{2}\right): f_{g}=0.99$, $0.9,0.5,0.1$ and 0.01 .

Figure 2. Final states dominance regions in $\left\langle\sigma_{a n n} v\right\rangle$, for $\tan \beta=8$ and $m_{h}=50 \mathrm{GeV}$. Sfermion masses are given by: $m_{\tilde{f}}=45 \mathrm{GeV}$, when $m_{\chi}<45 \mathrm{GeV} ; m_{\tilde{f}}=1.2 m_{\chi}$ otherwise (except for the SuSy partners of the top quark whose common mass is set at $\tilde{m}=3 \mathrm{TeV}$ ). Dominance region for a particular channel is defined as the region where that channel dominates over the other ones by a factor of five at least. Different regions are marked as follows: heavy dots for $f \bar{f}$ final state, horizontal lines for gauge boson pair final state, diamonds for mixed Higgs boson - gauge boson final state, squares for Higgs boson pairs final state. In regions marked with light dots, no dominance of a particular channel occurs.

Figure 3. Same as in Figure 2, with all sfermion masses fixed at $m_{\tilde{f}}=$ $3 \mathrm{TeV}$.

Figure 4. Scatter plots for neutralino relic abundance $\Omega_{\chi} h^{2}$ as a function of the neutralino mass $m_{\chi} . M_{2}$ and $\mu$ are varied in the ranges $20 \mathrm{GeV} \leq M_{2} \leq 6 \mathrm{TeV}$ and $20 \mathrm{GeV} \leq|\mu| \leq 3 \mathrm{TeV} ; \tan \beta$ is varied in the range $6 \leq \tan \beta \leq 10$; lightest scalar Higgs boson mass is $m_{h}=50 \mathrm{GeV}$; sfermion masses are taken as in Figure 2. (a) and (b) refer to neutralino compositions which are dominantly higgsino $\left(a_{1}^{2}+a_{2}^{2} \leq 0.1\right)$ or dominantly gaugino $\left(a_{1}^{2}+a_{2}^{2} \geq 0.9\right)$, respectively; (c) refers to the case when higgsino and gaugino components are maximally mixed $\left(0.45 \leq a_{1}^{2}+a_{2}^{2} \leq 0.55\right)$, for positive $\mu$; (d) the same as in (c), for negative $\mu$.

Figure 5. Same as in Figure 4(a and b), except for sfermion masses fixed at $m_{\tilde{f}}=3 \mathrm{TeV}$.

Figure 6. Scatter plots for neutralino relic abundance $\Omega_{\chi} h^{2}$ as a function of the neutralino mass $m_{\chi} . M_{2}$ and $\mu$ are varied in the ranges $20 \mathrm{GeV} \leq M_{2} \leq 6 \mathrm{TeV}$ and $20 \mathrm{GeV} \leq|\mu| \leq 3 \mathrm{TeV} ; \tan \beta$ is fixed at the value $\tan \beta=2$; lightest scalar Higgs boson mass is $m_{h}=80 \mathrm{GeV}$; sfermion 
masses are taken as in Figure 2. (a) and (b) refer to neutralino compositions which are dominantly higgsino $\left(a_{1}^{2}+a_{2}^{2} \leq 0.1\right)$ or dominantly gaugino $\left(a_{1}^{2}+a_{2}^{2} \geq 0.9\right)$, respectively; (c) refers to the case when higgsino and gaugino components are maximally mixed $\left(0.45 \leq a_{1}^{2}+a_{2}^{2} \leq 0.55\right)$, for positive $\mu$; (d) the same as in (c), for negative $\mu$.

Figure 7. Same as in Figure 6(a and b), except for sfermion masses fixed at $m_{\tilde{f}}=3 \mathrm{TeV}$.

Figure 8. Same as in Figure 6, except for $\tan \beta=20$ and $m_{h}=50 \mathrm{GeV}$. 
This figure "fig1-1.png" is available in "png" format from: http://arxiv.org/ps/hep-ph/9309218v1 
This figure "fig2-1.png" is available in "png" format from: http://arxiv.org/ps/hep-ph/9309218v1 
This figure "fig1-2.png" is available in "png" format from: http://arxiv.org/ps/hep-ph/9309218v1 
This figure "fig2-2.png" is available in "png" format from: http://arxiv.org/ps/hep-ph/9309218v1 
This figure "fig1-3.png" is available in "png" format from: http://arxiv.org/ps/hep-ph/9309218v1 
This figure "fig2-3.png" is available in "png" format from: http://arxiv.org/ps/hep-ph/9309218v1 
This figure "fig1-4.png" is available in "png" format from: http://arxiv.org/ps/hep-ph/9309218v1 
This figure "fig2-4.png" is available in "png" format from: http://arxiv.org/ps/hep-ph/9309218v1 
This figure "fig1-5.png" is available in "png" format from: http://arxiv.org/ps/hep-ph/9309218v1 
This figure "fig2-5.png" is available in "png" format from: http://arxiv.org/ps/hep-ph/9309218v1 
This figure "fig2-6.png" is available in "png" format from: http://arxiv.org/ps/hep-ph/9309218v1 